\title{
A Study of Redundancy of Time Dilation in Theory of Relativity
}

Devinder Kumar Dhiman*

G2Ocean, Damsgardsveien 165, Box 1925, 5160 Laksevag, Bergen, Norway; dhimandk@rediffmail.com

\begin{abstract}
Background/Objective: The great scientist of 20th century, Albert Einstein, through his Theory of Relativity, related time with space as a fourth dimension and introduced time dilation. The purpose of this article is to demonstrate the absolute nature of time. Method: The technique used is division of the space-time fabric into individual components of spacefabric and time and evaluating the effect of motion of a rigid body through the space-fabric. The effect is then assimilated in speed of light to establish the speed of light through the space-fabric while keeping the time invariable. Finding: It is found that the belief of variable time in the Theory of Relativity has its roots in variation of the speed of light due to the alteration of the space-fabric, when either a rigid body moves through it or there is a change of gravity. When this variation of the speed of light is incorporated in the proofs of the Theory of Relativity, such as the measurement of time dilation in the Hafele and Keating experiment, The Precession of the Perihelion of Mercury, The Bending of Starlight by the Sun and the measurement of time dilation by radio signals in traveling from Earth to Mars and back, it is found that they do not require variable time. This finding brings into the open the hidden factors which are instrumental in the adoption of time dilation. Application/Improvement: This finding has the potential to make the Theory of Relativity straightforward by eliminating the time paradox and further research on the space-fabric can help in understanding the source of gravity.
\end{abstract}

Keywords: Bending of Light, Caesium Atomic Clock, Perihelion of Mercury, Shapiro Delay, Space-time Fabric, Time Dilation, Theory of Relativity

\section{Introduction}

In the era of Galileo and Newton, it was observed that all the laws of mechanics are same for uniformly moving and stationary frames of reference. For instance, inside a smoothly sailing ship, a ball thrown upwards lands back in the hands of the thrower akin to a ball thrown upwards in a stationary frame of reference at shore. This happens due to the inertia gained by the ball when moving along with the ship. Any such experiment performed with the motion of the objects cannot be used in ascertaining whether the ship is moving or stationary, unless one hap- pens to look out of the port hole. This relation between stationary and uniformly moving frames of reference is known as the Law of Relativity.

When the same law is applied to light, it is found that the law does not hold good because light is massless and does not gain inertia. Because of this anomaly, it was felt that there was a need for modification of this law for adapting it to light as well.

In the year 1905, Special Theory of Relativity was proposed by Albert Einstein. This theory was valid for slow moving objects having inertia, as well as for the motion of the light. For formulating his theory, Einstein had to

${ }^{*}$ Author for correspondence 
make one significant assumption of having individual time for every object in contrast to the concept of universal time. This assumption led to the concept of time dilation for fast moving objects, where every uniformly moving object measures less time in comparison to the stationary object.

Special Theory of Relativity was restricted to the motion of uniformly moving objects. There was a further requisite for extending the theory to non-uniformly moving objects too, which was done by Einstein himself in the year 1915 by proposing the General Theory of Relativity.

The General Theory of Relativity articulates that the objects in gravitational field are comparable to objects having uniform acceleration. This theory revealed a relation between gravity, space and time, causing introduction of the concepts of space-time fabric and gravitational time dilation. Space-time fabric can be considered like a taut rubber sheet, which develops a curvature when a mass is placed on it. Anyplace where the space-time is curved, gravity gets enhanced and time slows down, causing gravitational time dilation.

In this article, we shall be discussing how both the Theories of Relativity can be harmonized with absolute time and hence, demonstrating the redundancy of time dilation due to uniformly moving objects, as well as gravity.

\section{Theories of Relativity}

Special Theory of Relativity ${ }^{1}$ is based on two postulates:

- The laws of physics are invariant in all inertial systems (non-accelerating frames of reference).

- The speed of light in a vacuum is the same for all observers, regardless of the motion of the light source.

The second postulate implies that every observer measures time individually, different from universal time. A moving observer measures time slower than a stationary observer.
According to the General Theory of Relativity:

- Space and time are related through a space-time continuum or fabric, which deforms in the presence of mass.

- Curvature of the space-time causes gravity, such that increased curvature results in higher gravity.

- Time slows down in the region of high gravity, causing gravitational time dilation 2 .

Thus, both the theories involve variation of time, however, we aim to study them without it. Therefore, we shall consider the space-time fabric bereft of the time component, i.e. we shall restrict our study to space-fabric only, with further restriction that it cannot penetrate rigid bodies.

Because we have not made any additional assumptions, we have only restricted them, there should not be any qualms in studying the effect of these restrictions, which we shall undertake theoretically, as well as mathematically.

\section{Motion of a Rigid Body through Space-fabric}

In accordance with our condition imposed on space-fabric, when a rigid body moves through it, it will behave similar to air in front of a moving train and get compressed. The compression will be maximum near the body and minimum at a distance sufficiently away from it as shown in Figure 1. This compression of space-fabric will affect the speed of light traveling through it.

Before we proceed further, we shall try to understand why the speed of light is considered constant.

From Maxwell's equations, we get the speed of light ${ }^{3}$, $c=\frac{1}{\sqrt{\mu \varepsilon}} \ldots(1)$, where $\varepsilon$ is electric permittivity and 
$\mu$ is magnetic permittivity. This formula suggests that

the speed of light is fixed as long as the electric permittivity and magnetic permittivity of space are fixed.

To understand these, we get help from the following two formulae:

Electrostatic force between two charges $q_{1}$ and $q_{2}$ placed at distance $r$, is given by the equation ${ }^{4}$

$$
E=\frac{q_{1} q_{2}}{4 \pi \varepsilon r^{2}}
$$

Therefore, if we consider two electrical charges of one Coulomb each, placed one meter apart, then the electric force between them will be $\frac{1}{4 \pi \varepsilon}$

Magnetic force between two conductors of equal length $\mathrm{L}$, placed at distance $r$, and carrying currents $\mathrm{I}_{\mathrm{A}}$ and $\mathrm{I}_{\mathrm{B}}$, is given by $F=B \times L$, where $\mathrm{B}$ is magnetic flux den-

sity.

Formula for magnetic flux density ${ }^{5}$ is $B=\frac{\mu I_{A} I_{B}}{2 \pi r}$

Therefore, $F=\frac{\mu I_{A} I_{B} L}{2 \pi r}$
If we consider two conductors of one meter each, place them one meter apart and pass an electric current of one Ampere through each of them, then the magnetic force between the two conductors will be $\frac{\mu}{2 \pi}$

From the above two statements, it can be noted that the ratio between electric force and magnetic force for equivalent charge and current, placed equal distance apart is:

$$
\frac{1 / 4 \pi \varepsilon}{\mu / 2 \pi}=\frac{1}{2 \mu \varepsilon}
$$

Comparing Equations 1 and 2, we note that the speed of light is equal to the square root of the double the ratio of electric and magnetic force generated in the space when a light signal travels through the magnetic and electric fields of the space and is constant only as long as this ratio remains unchanged.

Electric and magnetic forces in an electromagnetic wave act at right angles to each other and are produced simultaneously, similar to the forces produced in a rubber sheet and mass model of space-time fabric as shown in Figure 2.

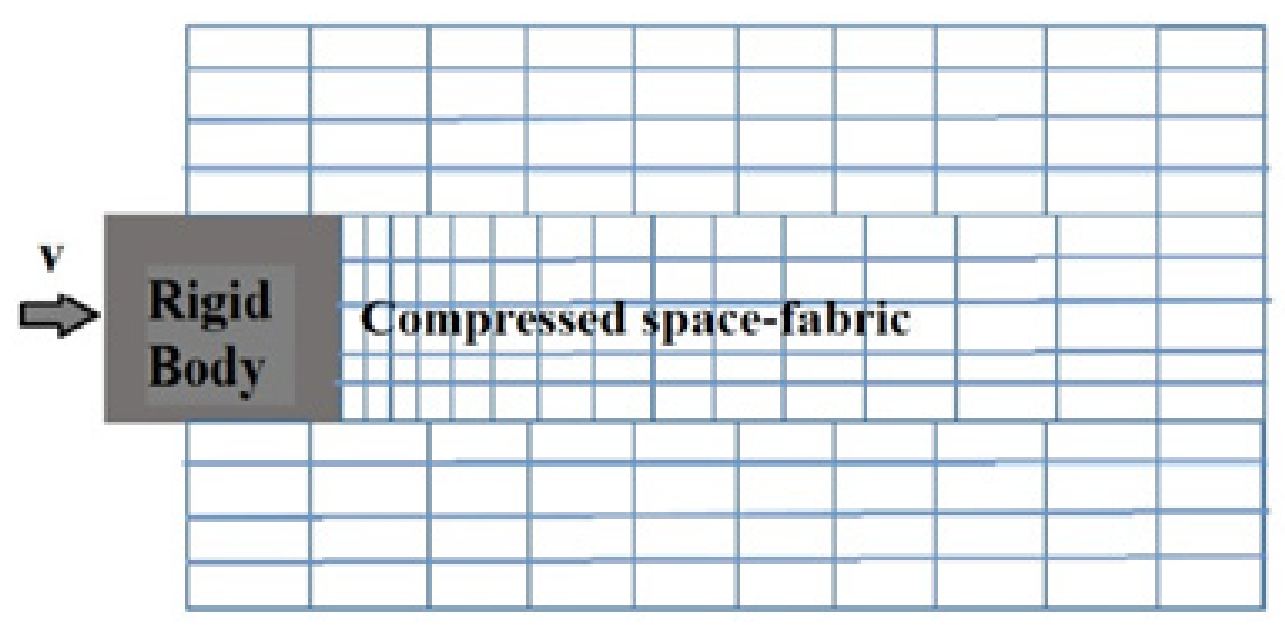

Figure 1. Rigid body moving through space-fabric. 


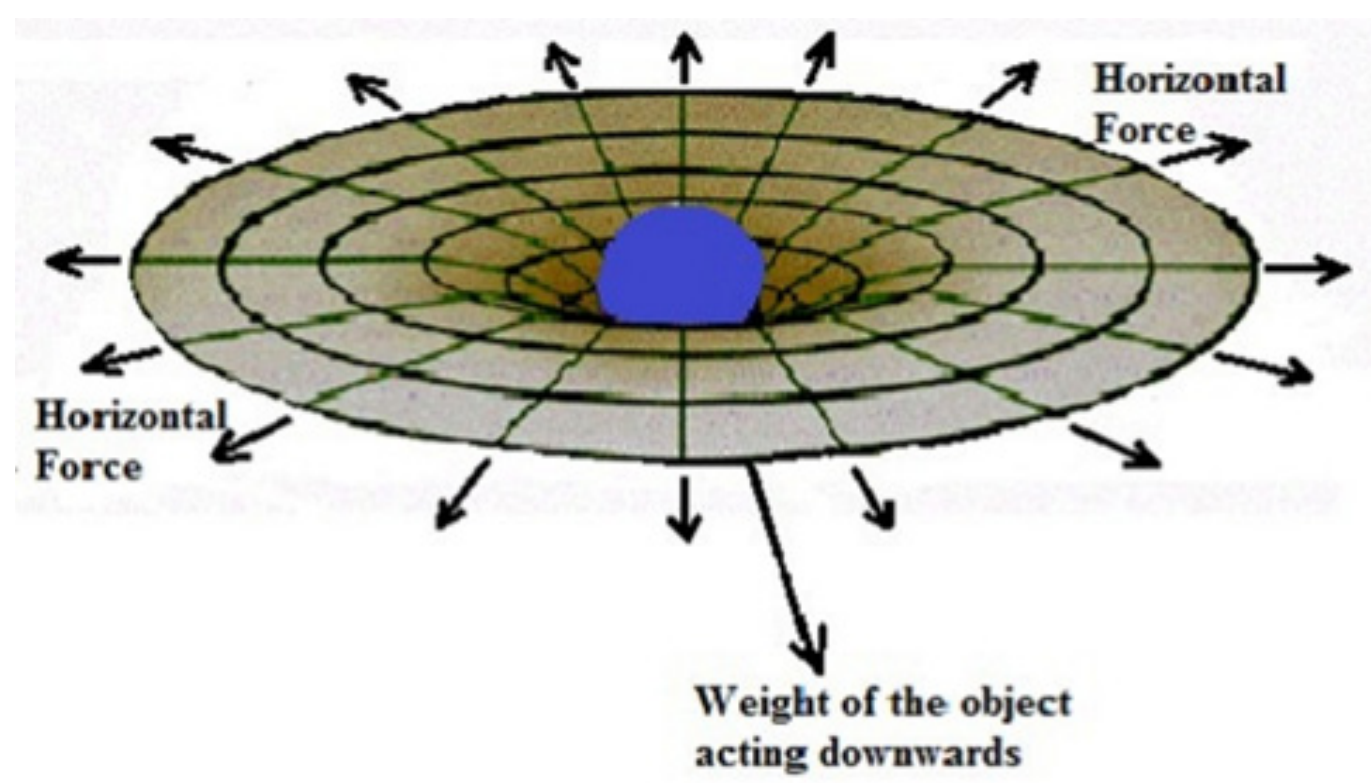

Figure 2. Perpendicular forces acting on rubber sheet when heavy mass is placed at its Center.

If the rubber sheet is held at its periphery on to a wall, there will be a force exerted on the wall when a heavy mass is placed at its center. This force will vary in accordance with the alteration of mass and will be horizontal, whereas the force due to mass is applied downwards. Thus, the two forces are perpendicular to each other and vary simultaneously in a set ratio.

We may also relate the scenario with a circus artist performing on a net tied to the walls. As the artist lands on the net, the net stretches and a horizontal force acts on the walls. Stretching the net gives back the push to the artist to jump again. As long as the elasticity and dimensions of the net remain unchanged, the artist gets back the same push everytime he/she lands on the net. But if the net becomes loose, the artist does not get the same push, indicating that the ratio of downward force and the perpendicular force has changed.

Similarly, magnetic and electric forces produced during transit of light through space-fabric will maintain a fixed ratio only as long as the space-fabric remains unchanged. With a change in the structure of space-fab- ric, the ratio between electric and magnetic forces must change similar to the effect of tightness of the net on the ratio of landing force of the artist and force exerted on the walls. So, we arrive at a situation where the speed of light traveling through the space-fabric, which is in front of a moving rigid body, will not be constant. This deduction is in line with an article published in Researchgate, titled "NASA's astonishing evidence that $\mathrm{c}$ is not constant: The Pioneer Anomaly

\section{Speed of Light Relative to Moving Rigid Body}

In the previous section we have noted that the speed of light differs with diverse compositions of the space-fabric. If we assume that the speed of light traveling through the compressed space-fabric is reduced by an amount equal to the speed of the rigid body itself, the relative speed of light with respect to the moving rigid body will remain equal to the relative speed of light with respect to stationary body. Similarly, in opposite case, where there is 
expansion of the space-fabric at the hind side of the moving rigid body, if the increase in speed of light is equal to the speed of the rigid body itself, then the relative speed of light with respect to the moving rigid body will remain equal to the relative speed of light with respect to stationary body.

Therefore, using the compression and expansion of space-fabric, we can show that the relative speed of light on either side of the moving rigid body can remain constant with respect to the moving rigid body and equal to the relative speed of light when the body was stationary. In other words, we can say that any moving or stationary observer, will measure the speed of light constant irrespective of his/her own motion without the use of time dilation. This constancy of the relative speed of light is in accordance with the Special Theory of Relativity.

\section{Lorentz's Transformations}

According to the Special Theory of Relativity, a co-ordinate system $K$ is considered corresponding to the

stationary frame of reference and another co-ordinate system $K^{\prime}$ corresponding to a moving frame of reference.

Then an event is described by three perpendicular coordinates of space and fourth co-ordinate of time in the stationary frame of reference $K$, and a moving frame of

reference $K^{\prime}$.

Let the values of the three perpendicular space coordinates in reference frame $K$ be denoted by $x, y, z$ and

time $t$. In moving frame of reference $K^{\prime}$, the same event will have corresponding values $x^{\prime}, y^{\prime}, z^{\prime}$, and $t^{\prime}$.Then the relation between corresponding values of two reference frames is given by Lorentz' $\mathrm{s}^{\underline{7}}$ equations:

$$
x^{\prime}=\frac{x-v t}{\sqrt{\left(1-v^{2} / c^{2}\right)}}
$$

$$
\begin{aligned}
& y^{\prime}=y \\
& z^{\prime}=z \\
& t^{\prime}=\frac{t-\frac{v x}{c^{2}}}{\sqrt{1-\frac{v^{2}}{c^{2}}}}, \text { where } \mathrm{v} \text { is the speed of the uniformly }
\end{aligned}
$$

moving rigid body.

Also, it may be noted that the values of $\mathrm{x}$ and $\mathrm{x}^{\prime}$ are given by $x=c t$ and $x^{\prime}=c t^{\prime}$ because $\mathrm{x}$ and $\mathrm{x}^{\prime}$ are the dis-

tances traveled by light in time $t$ and $t$ ' respectively on space-time co-ordinate scale, not the distances traveled by the moving body.

\section{Modified Transformations}

For variable speed of light, without time dilation, Lorentz's transformation equations will change.To find them, we shall keep $t$ constant and $C$ variable.

Again, let the values of three co-ordinates in stationary frame of reference $K$ in space-fabric be $x, y$ and $z$ at time $t$ and speed of light $C$. In moving frame of reference $K^{\prime}$, the co-ordinates will be $x^{\prime}, y^{\prime}$ and $z^{\prime}$ at time $t$ and speed of light $c^{\prime}$. Then Lorentz's equation for $x^{\prime}$ will 
become:

$$
x^{\prime}=\frac{x-v t}{\sqrt{1-\frac{v^{2}}{\left(c^{\prime}\right)^{2}}}}
$$

\section{Relation between c' and c}

In this case, distances traveled by light in time $t$ at speeds $c$ and $c^{\prime}$ will be given by $\mathrm{x}=\mathrm{ct}$ and $\mathrm{x}^{\prime}=\mathrm{c}^{\prime} \mathrm{t}$.

Substituting $x^{\prime}=c^{\prime} t$, and $x=c t$ in Equation 3, we

can write:

$$
c^{\prime} t=\frac{c t-v t}{\sqrt{1-\frac{v^{2}}{\left(c^{\prime}\right)^{2}}}}
$$$$
y^{\prime}=y,
$$

After dividing by $t$ on both sides, we get:

$$
z^{\prime}=z \text {, and }
$$

$$
c^{\prime}=\frac{c-v}{\sqrt{1-\frac{v^{2}}{\left(c^{\prime}\right)^{2}}}}
$$

$$
c^{\prime}=\sqrt{(c-v)^{2}+v^{2}}
$$

And after re-arranging, we arrive at the equation:

\section{Representation of c'}

$$
c^{\prime}=\sqrt{(c-v)^{2}+v^{2}}
$$

Substituting the value of c' from Equation 4 in We can represent $c^{\prime}=\sqrt{(c-v)^{2}+v^{2}}$ with hypotenuse of a right triangle as shown in Figure 3, where $c-v$ and Equation 3, we find:

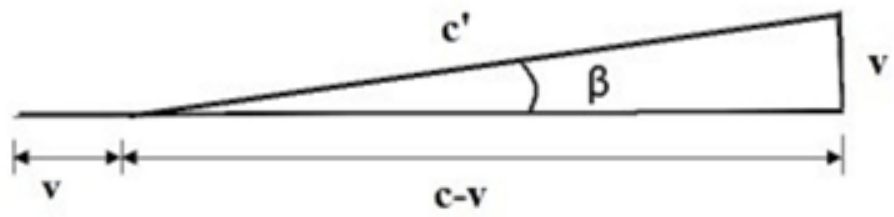

Figure 3. Representation of the speed of light. 
$v$ are other two sides. This diagram gives us an angle $\beta$ such that $\cos \beta=\frac{c-v}{c^{\prime}}$

\section{Increase of Distance Traveled by Light}

The distance traveled by light in time $t$ in front of moving

rigid bodyis $x^{\prime}=c^{\prime} t$ in the direction of $c^{\prime}$, as repre-

sented by line segment BE in Figure 4. Therefore, total distance covered by light and therigid body in time $t$ is $\mathrm{x}^{\prime}+\mathrm{vt}$

The distance traveled by light in time $t$ at speed $c$, in front of a stationary rigid body, is equal to $\mathrm{x}$.

Therefore, the difference in distance traveled by the combination of light wave andrigid body in the above two cases $=\left(x^{\prime}+v t\right)-x$

$$
=x^{\prime}-(x-v t) .
$$

Additional distance traveled by the light wave can be represented by DE in Figure 4, after we draw an arc CD such that $\mathrm{BC}=\mathrm{BD}$.
Since arc CD is infinitesimal, we can consider it to be a straight line, forming a side of the triangle CDE.

In triangle $\mathrm{CDE}$, angle $\mathrm{CDE}$ is nearly equal to $90^{\circ}$ as $\mathrm{CD}$ is very close to a tangent to arc $\mathrm{CD}$. That gives us the equality of angle DCE and DBC.

$$
\begin{aligned}
& \text { Thus, angle } \mathrm{DCE}=\beta \text {, and } \mathrm{DE}=\mathrm{CE} \sin \beta \\
& =\mathrm{v} \sin \beta \\
& =\mathrm{v}^{2} / \mathrm{c}
\end{aligned}
$$

This equation gives us the additional distance traveled by the light wave in one second that gets added to the path of light due to compression of the space fabric. We shall make use of this additional distance in further discussion on tests of the Relativity Theory.

\section{Gravitational Time Dilation}

Gravitational time dilation is given by the formula ${ }^{2}$

$$
\begin{aligned}
& t^{\prime}=t \sqrt{\left(1-\frac{2 G M}{r c^{2}}\right.} \\
& \text { Formula for escape velocity is } v_{e}=\sqrt{\frac{2 G M}{r}}
\end{aligned}
$$

where $G$ is gravitational constant, $M$ is mass of the

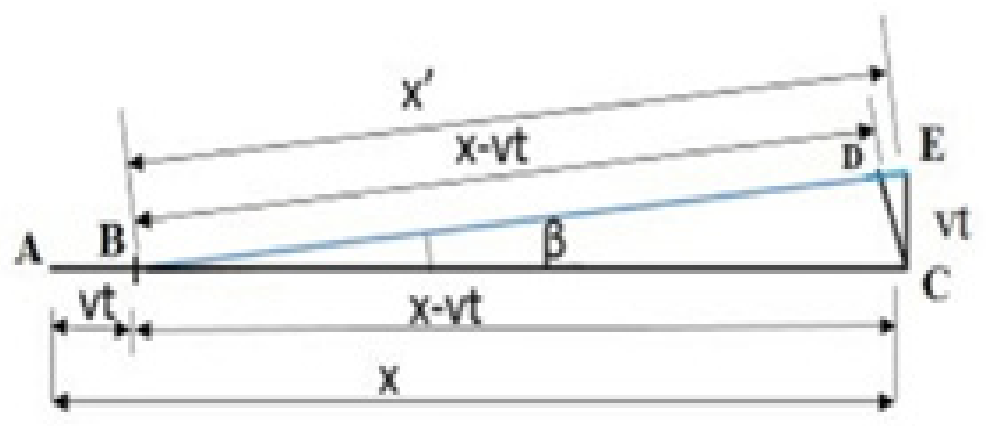

Figure 4. Increase in distance traveled by light due to compression of space-fabric. 
object causing gravity and $r$ is the distance from the cen-

ter of the object.

Substituting the value of $\frac{2 G M}{r}$ from Equation 8 in Equation 7, we get:

$$
t^{\prime}=t \sqrt{\left(1-\frac{v_{e}^{2}}{c^{2}}\right)}=\frac{t}{\gamma} \text {, showing that time dilation }
$$

caused due to a rigid body moving at escape velocity is nothing but gravitational time dilation ${ }^{2}$.

We have already noted that time dilation disappears in the Special Theory of Relativity if the speed of light is variable. For the same reason, gravitational time dilation vanishes as this is equivalent to the time dilation of a rigid body moving at a speed equal to the escape velocity of the rigid body at a distance $r$ from the center of the object causing the gravitational effect.

The above discussion implies that both the theories of Relativity can be adapted to absolute time.

However we note that both the theories claim time dilation and have strong verifications due to thefollowing observations:

- The precession of perihelion of Mercury.

- Bending of light by the Sun's gravity.

- Precise measurement of time dilation using Caesium atomic clocks by Hafele and Keating in 1971.

- Measurement of time in traveling of radio signals from Earth to Mars and back.

If we wish to harmonize both the theories with absolute time, then we must have an explanation for these phenomena also without the variation of time, which we shall discuss in the next sections.

\section{Precession of Perihelion of Mercury according to the General Theory of Relativity}

With every revolution of Mercury around the Sun, its closest point of approach to the Sun, known as perihelion, overshoots the perihelion of its previous orbit. The angle of advance of perihelion was measured 575 " for 100 earth years. Out of this, 532" could be accounted for by the gravitational pull of other planets, but the remaining 43" was a puzzle for scientists until 1915 when Einstein provided the rationale through his General Theory of Relativity.

The angle of advance of perihelion $\delta$, per orbit of Mercury $^{8}$, orbiting in a single plane, is given by the formula:

$$
\delta=\frac{6 \pi G M}{a\left(1-e^{2}\right) c^{2}}
$$

where M is the total mass of the Sun, a is the semimajor axis and $\mathrm{e}$ is the orbit's eccentricity.

\section{Precession of Perihelion using Variation of Space- fabric}

The revolution of Mercury around the Sun is three dimensional, but for simplicity, we shall consider the motion of Mercury in a single plane as shown in Figure 5.

Let us consider a position of Mercury at an angle $\phi$

from the center-line of the Sun and Mercury when Mercury is at its perihelion.Orbital velocity 9 of Mercury at a distance $r_{1}$ from Focus $F_{1}$ is given by:

$$
\left.v_{1}=\sqrt{G M\left(\frac{2}{r_{1}}\right.}-\frac{1}{a}\right)
$$

where $a$ is the semi-major axis. 
Distance of Mercury from Focus $F_{1}$ is given by

$$
r_{1}=a(1-e \cos \phi)
$$

Substituting the value of $r_{1}$ from Equation (10) in the

Equation (9) and rearranging the terms, we get:

$$
v_{1}=\sqrt{\frac{G M(1+e \cos \phi)}{a(1-e \cos \phi)}}
$$

When Mercury is traveling at velocity $v_{1}$ at point $A$

as shown in Figure 6, the space-fabric ahead of it gets deflected by a distance equal to $v_{1}$ over a distance trav-

eled by light in one second, as in the case for uniformly moving bodies shown in Figure 3.

After Mercury goes across the distance traveled by light in one second, it reaches point B and its direction of travel deviates by an angle $d \phi$ as shown in Figure 6.
Mercury, the space-fabric deflection will become the product of $v_{1}$ and the average change of direction of

Mercury in one second i.e. half of angle $d \phi$.

Therefore, the deflection of space-fabric over the distance traveled by light in one second and hence, the path of Mercury from its normal Newtonian pathfor point A in Figure 6,represented by $d s$ becomes:

$$
\begin{aligned}
& d s_{1}=\frac{v_{1} \times d \phi}{2} \\
& =\frac{v_{1} \times c_{1}{ }^{\prime}}{2 r_{1}} \\
& \text { (because } d \phi=\frac{c_{1}{ }^{\prime}}{r_{1}} \text { ) }
\end{aligned}
$$

Substituting the expression of $v_{1}$ and $r_{1}$ from Equations 9 and 10 in Equation 12, it becomes:

$$
d s_{1}=\frac{c_{1}{ }^{\prime}}{2 a(1-e \cos \phi)} \sqrt{\frac{G M(1+e \cos \phi)}{a(1-e \cos \phi)}}
$$

Considering this change of the direction of travel of

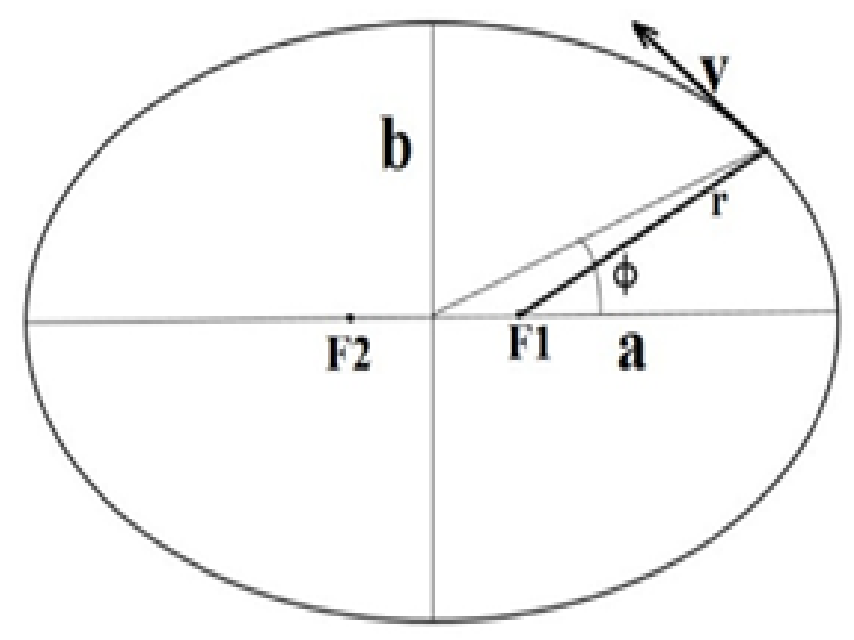

Figure 5. Elliptical path of Mercury moving at velocity ' $v$ ' when Sun is at focus F1. 


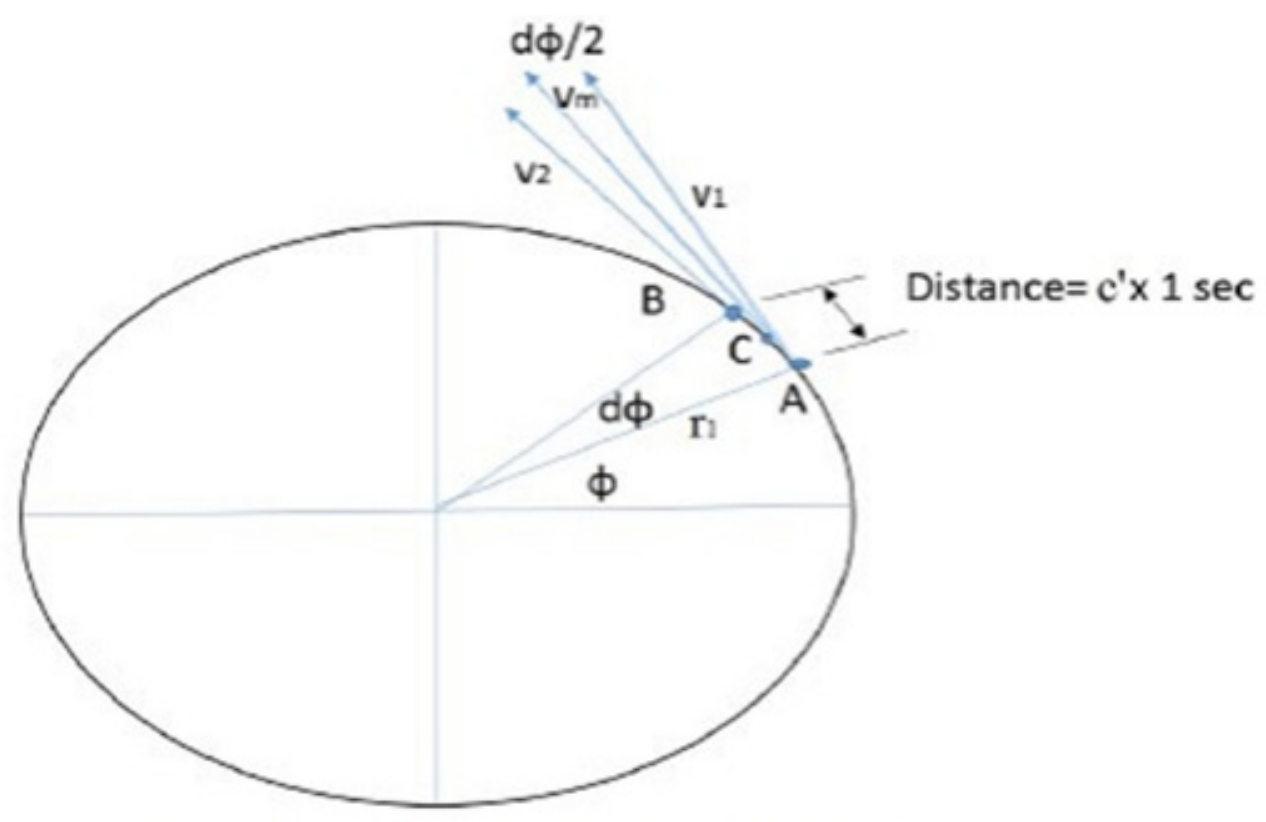

Figure 6. Change in direction of motion of Mercury over the distance traveled by light in one second.

This formula gives us the deflection of Mercury over a distance traveled by light in one second. This value changes with the increase of $\phi$ from 0 to 360 degrees in accordance with the velocity of the planet. By taking a summation of the deflection per second over the duration in which light traverses each quadrant, we can calculate the total deflection of Mercury from its normal path.

From Figure 7 we can note that $d S_{1}$ is the sum of all the deflections over the entire duration of travel of Mercury from 0 to 90 degrees and $\mathrm{dS}_{2}$ is for 90 to 180 degree.

Using Wolfram average calculator for Equation 13, we can find the average deflection of space-fabric per second in the first quadrant to be equal to $164.779 \mathrm{~m}$.

By dividing the length of arc of each quadrant by the speed of light we can calculate the time taken for light to travel through each quadrant. We find this value to be 299.782 second, therefore the total deflection of spacefabric and hence Mercury in the first quadrant will be:

$$
\sum_{t=0}^{t=299.782} \frac{c^{\prime} v}{2 r}=164.779 \times 299.782=49412.57 \mathrm{~m}
$$

The average angle of travel of Mercury in the first quadrant from its initial position to its final position is 45 degree to the major axis, therefore, the average deflection will also be at an angle of 45 degrees from major axis and hence, the component of the deflection of Mercury along the minor axis will be:

$$
49412.57 \times \operatorname{Sin} 45^{\circ}=34939.97 \mathrm{~m}
$$

Similarly, we can calculate the average deflection of the Mercury in the second quadrant using Wolfram average calculator for Equation 13. This value for the second quadrant is $=96.9757 \mathrm{~m}$. After multiplying it with total 
time taken by light to traverse the second quadrant, we get a total deflection:

$$
96.9757 \times 299.782=29071.569 \mathrm{~m} .
$$

Component of this deflection along the minor axis is:

$$
29071.569 \times \operatorname{Sin} 45^{\circ}=20562.88 \mathrm{~m}
$$

Two components of deflection for the first quadrant and the second quadrant will be opposite in direction, therefore, resultant will be the difference of two values, i.e.

$$
34939.97-20562.88=14377.09 \mathrm{~m}
$$

To calculate the angle of advance of Mercury in half cycle, we divide the net deflection of first two quadrants by the semi-major axis $=$

$$
\frac{14377.09}{57900000000}=2.48309 \times 10^{-7}
$$

(the semi major axis of Mercury's orbit around the Sun is $57900000000 \mathrm{~m}$ ).

For complete revolution of Mercury, the total angle of advance will be equal to twice this value, i.e.,

$$
\delta=2 \times 2.48309 \times 10^{-7}=4.96618 \times 10^{-7}
$$

Converting this angle into seconds for 100 earth years, taking one Mercury year as 87.97 Earth days, we get

$$
\delta=\frac{4.96618 \times 10^{-7} \times 100 \times 365.25 \times 3600 \times 180}{87.97 \times 3.14}=42.55^{\prime \prime}
$$

which is within the observed range of 42.5 to 43.5 seconds.

Thus, we have arrived at the observed value of the deflection of the path of Mercury without using Time Dilation. We have used only the deflection of space-fabric due to the motion of Mercury itself, which results in its own deflection as it passes through the deflected spacefabric.

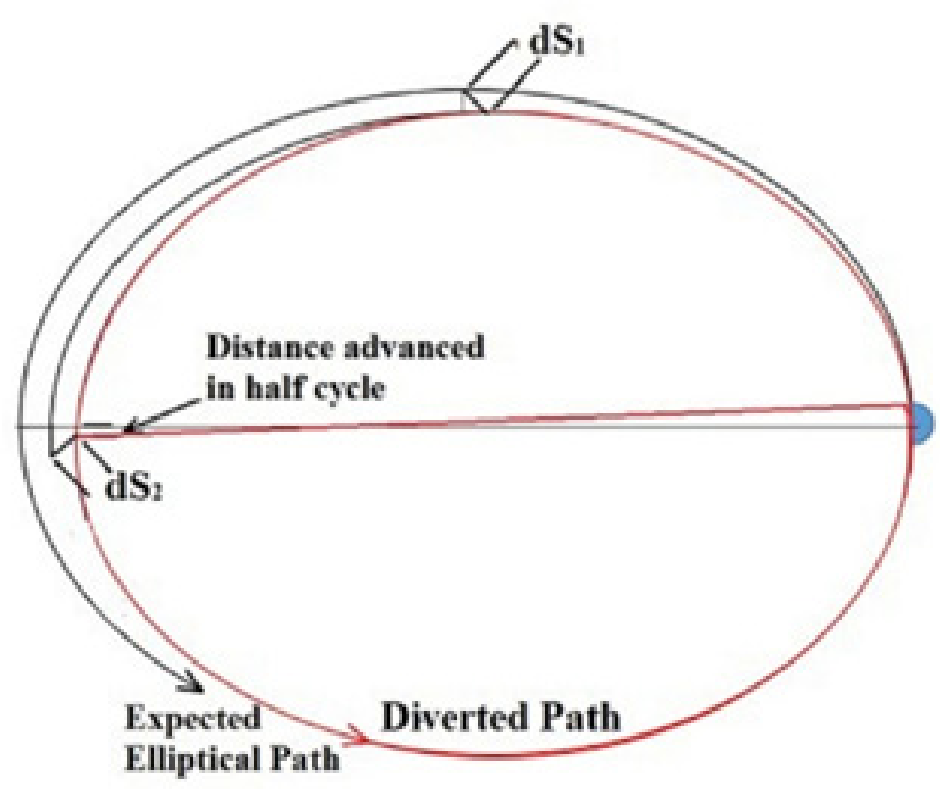

Figure 7. Advancement of perihelion of Mercury during its travel. 
It may be noted that the deflection of Mercury for the first half cycle along the major axis will be equal and opposite to the deflection of the second half cycle along the major axis and hence will cancel out.

\section{Bending of Light by Gravity}

When a ray of light coming from a distant star towards the Earth passes near the Sun, it bends by a small angle. Using Newtonian method, the angle of deviation was calculated 0.875 ", but that was not correct. Based on his General Theory of Relativity, Albert Einstein gave the formula $^{10}$ for calculating the angle of deviation of light as $\frac{4 G M}{b c^{2}}$, where $G$ is gravitational constant, $M$ is Mass of the body, $b$ is the nearest distance at which the light passes the Sun and $C$ is the speed of light. Value given by this formula was found correct through practical observations and it became an infallible proof for Einstein's General Theory of Relativity as well as Gravitational Time Dilation.

Notwithstanding this validation, we shall calculate the angle of deviation of light by the Sun using the formula utilized for the deviation of Mercury's path and ascertain whether it matches the observation or not.

\section{Bending of Light passing near the Sun without using Gravitational Time Dilation}

A rigid body at distance $b$ from the center of the Sun, has an orbital velocity equal to $v=\sqrt{\frac{G M}{b}}$. Space-fabric at that location in front of a moving rigid body will get deflected equal to the orbital velocity. Since the orbital velocity does not depend on the mass of the rigid body, a massless rigid body may be assumed to be traveling at a speed equal to orbital velocity at that location. Light passing in front of the massless rigid body through that location will get deflected by an amount equal to the orbital velocity $v$ due to deflection of space-fabric, in a direction perpendicular to the path of light as discussed in Section 4.

As light goes away from the Sun, the orbital velocity and its direction affecting the speed of light keeps changing as shown in Figure 8. It implies that the light has to pass through different concentrations of the space-fabric as it goes away from the Sun, which causes light to deflect differently. The amount of deflection is given by the formula $\frac{c^{\prime} v}{2 r}$, which is same as for the deflection of Mercury.

Orbital velocity of the imaginary massless rigid body pushing the space-fabric at distance $r_{1}$ is

$$
v_{1}=\sqrt{\frac{G M}{r_{1}}}
$$

Then, from the diagram 8 ,

$$
r_{1}=\sqrt{\left(b^{2}+\left(c^{\prime} t\right)^{2}\right.} \text { and } \cos \phi=\frac{b}{r_{1}}
$$

Light travels for one second to reach another point at a distance $r_{2}$ from the center of the Sun. Orbital velocity at that point will be $v_{2}$. Then the angle between $v_{1}$ and $v_{2}$ will be approximately given by $d \phi=\frac{c^{\prime} \cos \phi}{r_{1}}$

The horizontal component of $\mathrm{v}_{1}$ in the direction of the travel of light will be $v_{1} \cos \phi$. 
Deviation of space-fabric at distance $r_{1}$ can be found using the formula given in Equation 12 as

$$
\frac{c^{\prime} v_{1} \cos ^{2} \phi}{2 r_{1}}
$$

Substituting the values $v_{1}=\sqrt{\frac{G M}{r_{1}}}$,

and $\cos \phi=\frac{b}{r_{1}}$ in the above equation,

we get deviation $=\frac{c^{\prime} b^{2} \sqrt{G M}}{2 r_{1}^{3.5}}$

Due to the variation of distance from the Sun aslight travels, we require an additional factor $\sqrt{\frac{B}{r_{1}}}$

where $B$ is the radius of the Sun and $r_{1}$ is the distance of light wave from the Sun at the instant $t_{1}$.

Thus, the deflection of light at radius $r_{1}$ (due to the deviation in space-fabric) becomes

$$
\frac{c^{\prime} b^{2} \sqrt{B(G M)}}{2 r_{1}^{4}}
$$

The above formula gives the deviation in the space fabric due to the horizontal component of orbital velocity, as seen in Figure 8 at any instant. The total deviation of the space-fabric due to the horizontal component of orbital velocity $v$ is:

$$
\Delta \mathrm{H}=\sum_{t=0}^{t} \frac{c^{\prime} b^{2} \sqrt{B} \sqrt{G M}}{2\left(r_{1}\right)^{4}}
$$

After substituting $r_{1}=\sqrt{\left(b^{2}+\left(c^{\prime} t\right)^{2}\right.}$ in above equation, we get:

$$
\Delta_{\mathrm{H}}=\sum_{t=0}^{t} \frac{c^{\prime} b^{2} \sqrt{B} \sqrt{G M}}{2\left(b^{2}+(c t)^{2}\right)^{2}}
$$

where $t$ is the time taken by light to travel from the Sun to the Earth.

In this formula, we shall substitute $c$ in place of c' to simplify the formula because the variation in speed of light at every interval is very small

$$
\Delta_{\mathrm{H}}=\sum_{t=0}^{t} \frac{c b^{2} \sqrt{B} \sqrt{G M}}{2\left(b^{2}+(c t)^{2}\right)^{2}}
$$

After taking out all constant terms of Equation 17 before the summation, we get:

$$
\Delta_{\mathrm{H}}=\frac{b^{2} \sqrt{B} \sqrt{G M}}{2 c^{3}} \sum_{t=0}^{t=498.667} \frac{1}{\left((b / c)^{2}+t^{2}\right)^{2}}
$$

Value of $\frac{b^{2} \sqrt{B} \sqrt{G M}}{2 c^{3}}$ is 2726372.272

$$
\text { And value of } \sum_{t=0}^{t=498.667} \frac{1}{\left((b / c)^{2}+t^{2}\right)^{2}} \text { for } \mathrm{t}=0 \text { to }
$$

498.667 seconds, when light just passes near the surface of the Sun, is found using Wolfram Summation Calculator to be equal to 0.0628964 .

Therefore, the total deflection of light due to horizontal component of velocity, (perpendicular to the path of light because deviation due to space fabric is perpendicular to the direction of travel of light), becomes:

$$
\begin{aligned}
& \alpha_{\mathrm{H}}=\frac{\sqrt{B} \sqrt{G M}}{2 c} \sum_{t=0}^{t=498.667} \frac{1}{\left((b / c)^{2}+t^{2}\right)}=2726372.272 \\
& \mathrm{x} 0.0628964=171479 \mathrm{~m}
\end{aligned}
$$




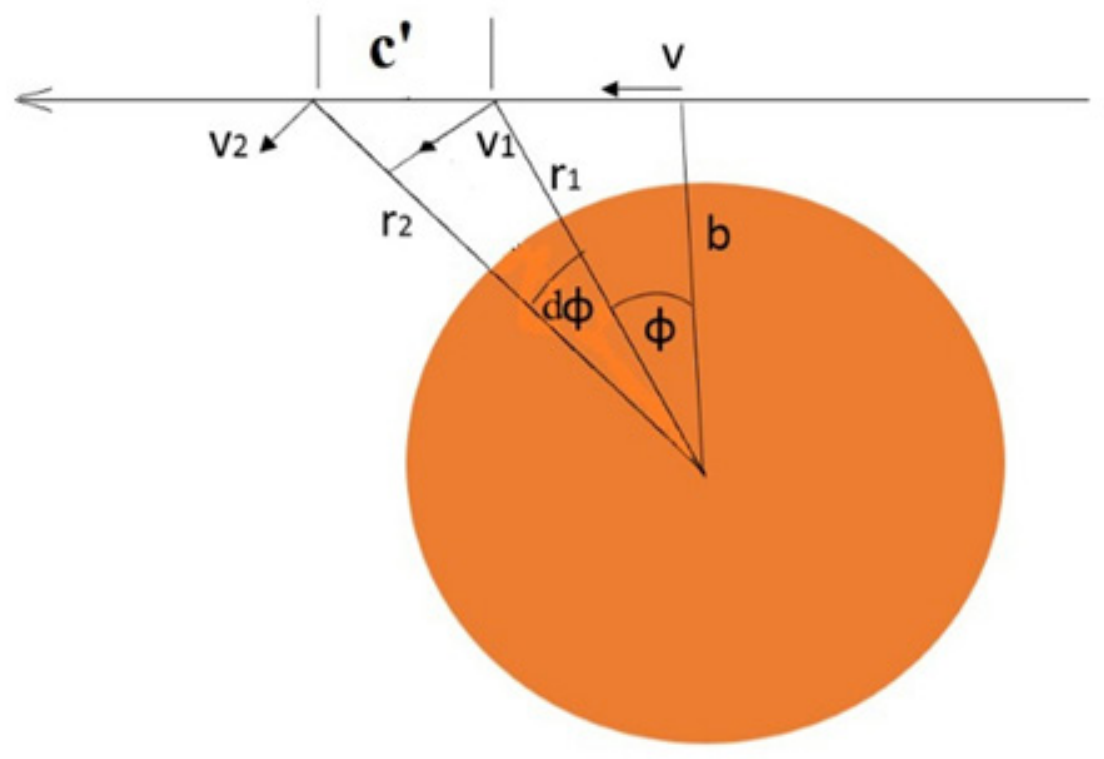

Figure 8. Orbital velocities in the path of light at two points apart by a distance equal to c'.

The effect of deviation in the space-fabric due to the vertical component of orbital velocity $\mathrm{v}_{1}$ on the path of light will be zero. However, there will be a complete shift of the space-fabric along the vertical component of orbital velocity $\mathrm{v}_{1}$ and will be equal to $\mathrm{v}_{1} \sin \phi$.

Multiplying it with $d \phi / 2$ and $\sqrt{\frac{B}{r_{1}}}$ as was done in the case of the horizontal component, we get the vertical deflection:

$$
\Delta_{\mathrm{V}}=\frac{c v_{1} \sin \phi \cos \phi \sqrt{B}}{2 r_{1}^{1.5}}
$$

After substituting the values of $v_{1}=\sqrt{\frac{G M}{r_{1}}}$ in Equation 18, we obtain:

$$
\Delta_{\mathrm{v}}=\frac{c \cos \phi \sqrt{B} \sqrt{G M}}{2 r_{1}^{2}} \times\left(1-\cos ^{2} \phi\right)
$$

After plugging value of $\cos \phi$ and $r_{1}=\sqrt{\left(b^{2}+\left(c^{\prime} t\right)^{2}\right.}$ in the above equation, we have:

$$
=\frac{c b \sqrt{B} \sqrt{G M}}{2\left(b^{2}+\left(c^{\prime} t\right)^{2}\right)^{1.5}} \times\left(1-\frac{b^{2}}{b^{2}+\left(c^{\prime} t\right)^{2}}\right)
$$


After re-arranging and replacing c' with $\mathrm{c}$ (due to the negligible difference between $c$ and $c^{\prime}$ ), we have:

$$
\Delta_{\mathrm{V}}=\frac{(c t)^{2} c b \sqrt{B} \sqrt{G M}}{\left(b^{2}+(c t)^{2}\right)^{2.5}}=\frac{t^{2} b \sqrt{B} \sqrt{G M}}{c^{2}\left((b / c)^{2}+t^{2}\right)^{2.5}}
$$

The total deflection due to the shift in the space-fabric caused by the vertical component of orbital velocities for the entire path of light travel from the Sun to the Earth in time 0 to 498.667 seconds can be obtained by taking the summation of all the deflections calculated above as:

$$
\alpha_{\mathrm{v}}=\sum_{0}^{498.667} \frac{t^{2} b \sqrt{B} \sqrt{G M}}{c^{2}\left((b / c)^{2}+t^{2}\right)^{2.5}}
$$

After separating the constants from the summation, we get:

$$
\alpha_{\mathrm{v}}=\frac{b \sqrt{B(G M)}}{c^{2}} \sum_{t=0}^{t=498.667} \frac{t^{2}}{(b / c)^{2}+t^{2}}
$$

Value of $\frac{b \sqrt{B(G M)}}{c^{2}}=2350320.924$

$$
\text { Value of } \sum_{t=0}^{t=498.667} \frac{t^{2}}{(b / c)^{2}+t^{2}}=0.0619282 \text {, as obtained }
$$

using Wolfram Summation calculator/

Therefore, the deflection due to a shift of space-fabric by the vertical component of orbital velocities will be equal to $2350320.924 \times 0.0619282=145551.144 \mathrm{~m}$.

Deflection due to the horizontal and the vertical components of velocity are caused due to two different reasons but both are in the direction perpendicular to the propagation of light, therefore they can be added and

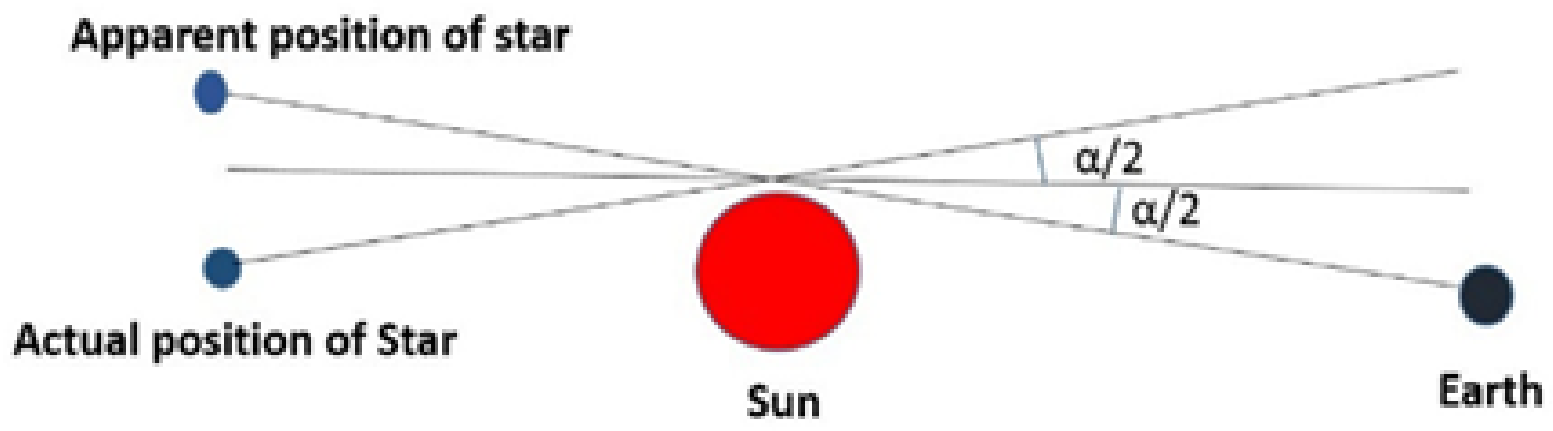

Figure 9. Angle of deviation of star light when passing near the Sun. 
the addition of both the deflections is $171479+145551=$ 317030.

Dividing the total deflection by the distance between the Sun and Earth (149600000000 m) we get the average angle of deflection $=2.11919 \times 10^{-6}$ radian .

Converting this angle to seconds, we get the value of the deflection angle equal to 0.437335 ".

After multiplying it by 2 because the total deflection is twice the angle of deflection measured as seen in Figure 9 we get $\alpha_{s}=0.87467$ " due to alteration in space fabric.

Gravitational deflection: Gravitational deflection of light can be calculated by the formula ${ }^{11}$

$$
a_{G}=\frac{2 G M}{c^{2} b}, \text { which is } 0.87445 ”
$$

Thus, the total deflection of light traveling from the Sun to the Earth can be obtained by the sum of two deflections, i.e. Gravitational deflection + deflection due to space fabric $=\alpha_{G}+\alpha_{S}=0.87445+0.87467=1.74912 ”$
The deflection calculated using the formula obtained from the Theory of Relativity is 1.7489 ", which is nearly equal to the value obtained by us. This equality demonstrates that the bending of light by gravity does not require space-time fabric, it can be explained with spacefabric only.

\section{Time Dilation Measured by Caesium Atomic Clock}

In a Caesium atomic $\operatorname{clock}^{\underline{12}}$, as shown in Figure 10. Caesium is first heated in an oven. Caesium atoms boil off and pass through a magnetic field during their travel through a tube maintained at a high vacuum. The magnetic field selects atoms of the right energy state which pass through a microwave field. The frequency of the microwave energy maintains 9,192,631,770 Hertz. The frequency varies slightly up and down, such that in each

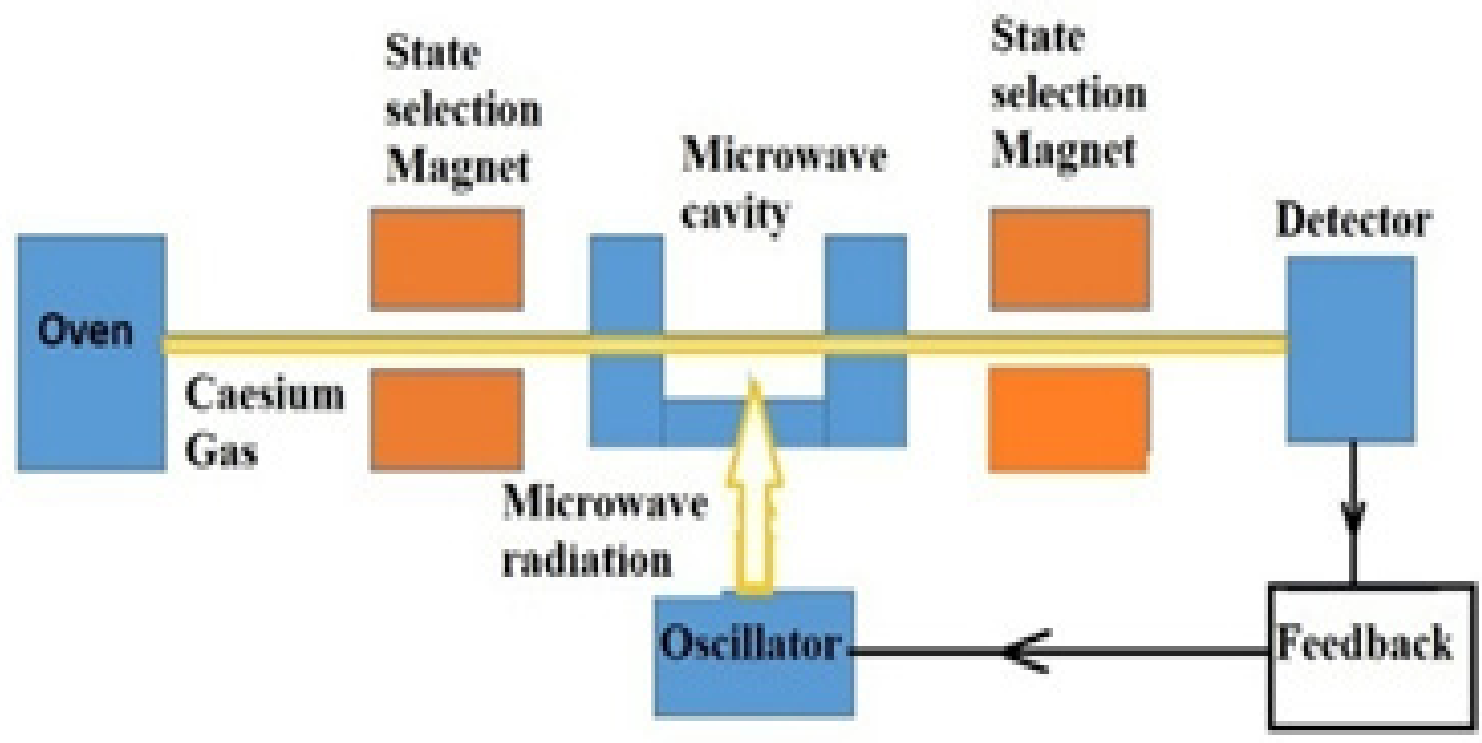

Figure 10. Working principle of Caesium atomic clock 
variation, it crosses the set frequency. Microwave generator is an accurate electronic oscillator to maintain a close range. When a Caesium atom receives microwave energy at exactly the right frequency, it changes its energy state.

Atoms pass through another magnetic field, which separates out the atoms that have changed their energy state. At the end of the tube, they strike a detector which gives an output proportional to the number of atoms striking it and its output is maximum when the microwave frequency is exactly correct. This information is used to make the slight correction necessary to bring the oscillator and the microwave field exactly on frequency and the frequency gets locked there. This locked frequency is then divided by $9,192,631,770$ to give the one pulse per second to measure time.

\section{Effect of Alteration in the Space-fabric on Time Measured by Caesium Atomic Clock}

When the Caesium atomic clock is taken on a flight, it acquires the velocity of the plane, and as we have noted in Equation 3, the distance traveled by light in the moving frame of reference in time $t$ changes from $x$ to $x^{\prime}$. The

microwave radiations emitted from the oscillator will then get affected by this change, such that the wavelength of the radiation will be shortened because $x^{\prime}$ is less than

$x$. Speed of the microwave radiation will also reduce to

$c^{\prime}$ from $c$, though its relative speed with respect to Caesium atoms will remain constant, i.e., equal to $c$ as we have noted in Section 4. The net effect of this change will be to increase the frequency of the microwave radiation hitting the Caesium atoms.

The number of atoms having the exact frequency reaching the end of the tube will change and due to the feedback given to the oscillator, lower frequency microwave radiations will be generated until the clock gets stabilized again with a new locked frequency. Measurement of the new locked frequency, which will be lower than the locked frequency of the stationary Caesium clock, will result in recording less time, which is considered validation of time dilation, whereas this is the effect of motion of the Caesium atomic clock due to its dependency on microwave radiations for measuring time.

\section{Traveling of Radio Signal from Earth to Mars and BackT}

In 1964, Irwin Shapiro published a paper titled 'Fourth test of General Relativity" and predicted that a radio signal going from Earth to Mars and back should be delayed by about 200 microseconds over the time calculated from Newtonian considerations. This delay was named the Shapiro delay. On Nov $26^{\text {th }}, 1976$, Mars, as seen from Earth, just grazed the southern limb of the Sun. At that time, measurements were made using project Viking Landers and enough data was collected to confirm this delay of approx. 250 micro second $\frac{13}{}$.

We can calculate this delay by our technique of reduction of the speed of light due to the motion of a rigid body. As the radio signal goes from Earth towards the Sun, it enters higher gravitational effect and the orbital velocity keeps changing. Effect of a massless rigid body traveling at orbital velocity on the speed of radio signal is such that $c^{\prime}=\sqrt{(c-v)^{2}+v^{2}}$.

From Equation 6, we know that the increase in distance traveled by light due to the compression of 
space-fabric in front of a rigid body, corresponding to a deflection $\mathrm{v}$ is given by $\mathrm{v}^{2} / \mathrm{c}$. Besides the deflection due to the compression of space fabric, there is equal deflection of light due to gravitation and increase of distance is $\mathrm{v}^{2} / \mathrm{c}$. Therefore, the total increase in distance traveled by light becomes $2 \mathrm{v}^{2} / \mathrm{c}$.

For radio signal traveling from the Earth to the Sun and back, the increase in distance will be given by $\sum_{-498.667}^{+498.667} \frac{2 v^{2}}{c}$, because time taken for one way travel is 498.667 second.

Using Wolfram summation, we can calculate its value to be equal to $35783.4 \mathrm{~m}$

Similarly, for the path of radio signal from Mars to the Sun and back to Mars (759.667 seconds for one way travel), we can calculate the increase in distance as $38287.06 \mathrm{~m}$

The total increase in the path of a radio signal from the Earth to Mars and back is equal to $35783.4+38287.06$ $=74070.46 \mathrm{~m}$.

Dividing this distance by the speed of light, we get time 0.0002469 second, i.e. 246.9 micro second, which is equal to Shapiro delay measured in 1976 in the Viking experiment. Hence, Shapiro delay, the fourth test of relativity, can also be explained without time dilation.

\section{Conclusion}

We have noted through the illustration of a rubber sheet and heavy mass that it is possible that the speed of light can vary because it depends on the ratio of electric and magnetic fields of the space through which it travels, and this ratio changes in the vicinity of moving rigid bodies and under the influence of gravitational field. We have also noted during the discussion in this article that one formula developed using the compression of the space- fabric due to the velocity of a rigid body, explains both the events of precession of perihelion of Mercury and bending of light by the Sun. Also, we have noted that Shapiro delay is the effect of change of speed of light due to compression of the space-fabric and gravitational deflection. We have also discussed how the time dilation might have got verified by practical measuring through Caesium atomic clocks in spite of there being no time dilation.

Our approach of finding the value of deflection in the path of Mercury and bending of light passing near the Sun, without time dilation, helps in avoiding the twin paradox and time travel speculations. We conclude that time is absolute and every phenomenon that uses time dilation can be explained using the concept of space-fabric as elucidated in this article, making time dilation redundant. Hence, we have demonstrated that both the theories of Relativity can be harmonized with absolute time if the speed of light is not constrained to be constant throughout the universe.

\section{References}

1. Pais A. Subtle is the Lord - The science and the life of Albert Einstein. Great Britain: Oxford University Press; 1982. p. 140.

2. Collier P. A most incomprehensible thing - Notes towards a very gentle Introduction to the mathematics of Relativity. 2nd ed. Germany: Incomprehensible Books; 2014. p. 20911. PMid: 24549970 PMCid: PMC4040150.

3. Khan MS. Foundation of Theory of Everything: Nonliving and Living Things. Indian Journal of Science and Technology. 2010; 3(9):955-81.

4. Coulomb's Law. 2014. http://hyperphysics.phy-astr.gsu.edu/ hbase/electric/elefor.html

5. Theory of Electromagnetic Fields. 2014. https://arxiv.org/ pdf/1111.4354.pdf

6. NASA's astonishing evidence that $c$ is not constant: The pioneer anomaly. 2016. https://www.researchgate.net/publication/2178125

7. Lorentz transformations. 2015. http://hyperphysics.phy astr. gsu.edu/hbase/Relativ/ltrans.html\#c2 
8. A Detailed Classical Description of the Advance of the Perihelion of Mercury. 2016. http://www.newtonphysics. on.ca/mercury/

9. Orbital Mechanics Course Notes. 2017. http://kestrel.nmt. edu/ dwestpfa/courses/notes.pdf

10. Nyambuya GG, Simango W. On the Gravitational Bending of Light. International Journal of Astronomy and Astrophysics. 2014; 4:250-63. Crossref.
11. Estimating light bending using order-of-magnitude physics. 2016. http://web.mit.edu/sanjoy/www/cv.pdf

12. The Brazilian time and frequency atomic standards program. 2016. http://www.scielo.br/scielo.php

13. Reasenberg RD, Shapiro II, MacNeil PE, Goldstein RB, Breidenthal JC, Brenkle JP. Viking relativity experiment - Verification of signal retardation by solar gravity. Astrophysical Journal. 1979; 234:219-21. Crossref. 